Final Report

DOE Report No.

DOE/ER/13243-8

Title

\title{
MULTILEVEL RELAXATION PHENOMENA AND POPULATION TRAPPING
}

Report Period

July 1, 1984 - June 30, 1991

Personal Author

F. T. Hioe

Grantee's Name

St. John Fisher College

and Address

Rochester, New York 14618

Report Date

November 1991

Prepared for

DOE Sponsorship and

The U.S. Department of Energy

DOE Instrument Number

Agreement No. DE-FG02-84ER13243

DISCLAIMER

\begin{abstract}
This report was prepared as an account of work sponsored by an agency of the Linited States Governinent. Neither the United States Government nor any agency therenl, nor any of their employees, makes any warranty, express or implied, or arsumes any legal liability of responsibility for the accuracy, completeness, or usefulness of any information, apparatus, product, or process discilosed, or represents that its use would not irfringe privately owned rights. Reference herein to any specific commercial product, process, or service by trade name, tradematk, manufacturer, or otherwise does not necessarily constitute or imply its endorsement, recommendation, of favoring by the United States Government or any agency thereor. The view's and opinions of authors expressed herein do not necessarily state or reflect those of the United States Government or any agency thereof.
\end{abstract}




\section{Abstract}

This final report summarizes the main results of our work supported by DOE since 1982. A list of 45 publications supported by this DOE Grant is attached at the end of this report.

The use and exploitation of the $S U(N)$ dynamic symmetry to the study of the dynamics of laser-atom interaction was the starting point of our research work under this DOE Grant, and is our most original contribution to the field of quantum electrodynamics. Many results of general and special interests have been derived and developed from this starting point and the following is a summary of them:

(1) We have introduced a set of simple relations based on the principle of unitary invariance which has proved to be useful for the study of the dynamics of a quantum system involving arbitrary coupling.

(2) We have found various specific conditions under which (a) we may have trapped population, or (b) we may send laser pulses through a multilevel atomic medium without attenuation.

(3) We have found a remarkably efficient method for optimal state selective multiphoton population transfer, that employs two or more spatially overlapping lasers arranged in an unconventional sequence which we called "counterintuitive". A recent suggestion by Profs. P. Marte, P. Zoller and J.L. Hall to use this counterintuitive method for atomic beam deflections promises to make this remarkably effective procedure to become an important method in atomic interferometry. 
The starting point of this DOE supported research project entitled "Multilevel Relaxation Phenomena and Population Trapping" was a discovery by the author and Prof. J. H. Eberly of a set of simple and useful conservation laws in the dynamics of interaction between lasers and multilevel atoms which hold true for any arbitrary coupling or laser intensity. The conservation laws are a consequence of unitarity in the $S U(N)$ croup, and we called the result as one arising from the SU(N) d,namic symmetry. These simple conservation laws can be simply used to deduce various restrictions in the population distribution among the energy levels when an atom interacts coherently with a laser. They also serve generally as a useful check for numerical calculations. More importantly, the idea of using dynamic symmetry to the problem of multiphoton excitation of multilevel atomic systems has produced many other fruitfu! ideas which have led to many unexpected and practically useful results.

\section{Adiabatic Following in Multilevel Systems}

We were the first to use the $S U(N)$ coherence vector picture for a generalized interpretation of the adiabatic following process for the problem of achieving population inversion in a multilevel system using one or more lasers whose detunings or/and amplitudes are continuously varied. We showed that, unlike an $\mathrm{N}=2$ level system, for a multilevel $(N>2)$ system, the $\left(N^{2}-1\right)$-dimensional $S U(N)$ coherence vector $S(t)$ does not and cannot be made to closely follow the field vector $\Gamma(t)$ in a quasisteady process that characterizes adiabatic following. However, the coherence vector $S(t)$ can be confined to move in a subspace spanned by an appropriately constructed (N-1) time-dependent field vectors, $\Gamma_{1}(t), \Gamma_{2}(t), \ldots, \Gamma_{N-1}(t)$. Thus only for the case of $N=2$ the picture of $S(t)$ "following" the field vector $\Gamma(t)=$ $\Gamma_{1}(t)$ emerges.

This understanding of generalized adiabatic following process led us to

(a) a simple analytic result which clearly illustrates how the populations and coherences evolve at all time, and

(b) suggest a way of achieving complete transfer of population from state 1 to state $\mathrm{N}$ by continuously changing the detunings of the incident laser in an "unconventional" interaction sequence which we 
called "counterintuitive".

For example, result (b) above suggests that efficient population transfer in the case of $N$ levels of decreasing spacings involving stepwise transitions such as in the case of levels in hydrogen atoms, should be accomplished by tuning the frequency of the incident laser pulse, which can be done by appropriately Stark shifting the levels by a varying electric field, in such a way that the interaction is first between the uppermost pair of levels and last with the lowermost one, even though the population is initially entirely in the lowermost level. Result (a) enabled us to see another distinctive and advantageous feature of the multilevel adiabatic following process: the intermediate levels are never significantly populated during the process, making the process insensitive to the possible short decay times of the intermediate levels. Another hidden suggestion, to be discussed in item 3 of this report, is an important extension of this "counterintuitive" interaction sequence to the case of sending two or more overlapping laser pulses for the problem of state-selective excitation process.

2. Conditions for Population Trapping, Complete Population Transfer and for Multiple Optical Soliton Propagation

An important idea which we have made good use of in our group theoretical consideration of the $S U(N)$ symmetry is the concept that different realizations of the same group can be employed for the same dynamical systems under different experimental conditions. This led us to the precise formulation of the conditions under which a system of $\mathrm{N}$-level atoms interacting with one or multiple lasers exhibits what we called

(a) a Gell-Mann dynamic symmetry, or

(b) an SU(2) or Cook-Shore dynamic symmetry,

or else. The conditions under which the systems exhibiting a GellMann or an $\mathrm{SU}(2)$ symmetry would permit a lossless propagation of multiple simultaneous optical solitons through the atomic media without attenuation have been obtained.

A lossless soliton propagation generally requires several conditions: For the $\mathrm{N}$ dipole-connected energy levels of each atom of 
the atomic medium, the dipole moments have to satisfy certain relations, the atomic medium must be partially excited out of its ground state initially, and the pulse amplitudes have to satisfy appropriate relations. In addition to the known hyperbolic-secant pulse shapes which are found to be appropriate for some specific conditions, we have discovered a whole new set of "higher order" soliton pulse shapes which are appropriate for other conditions. These theoretical results of ours presently still await experimental verifications, and so do their practical applications.

We have also found many less restrictive and more easily realizable conditions, than the ones for the Gell-Mann or SU(2) type symmetry, for an atomic system interacting with lasers to exhibit population trapping. We have extended the concept of population trapping to one that is generally characterized by a set of "constants of evolution".

We have also derived and accumulated a good collection of analytic results for 2-, 3- and $\mathrm{N}$-level time-dependent excitation problem for various types of pulse shapes and detunings. These are non-adiabatic results which complement the adiabatic following results in items 1 and 3 of this report, and they are very instructive in showing how the adiabatic following limits are approached. One of the most important uses of these results is the understanding of the conditions under which a complete transfer of population from one state to another can be achieved, and the understanding of how and why certain population distribution is torbidden.

\section{Optimal Population Transfer by Counterintuitive Pulse Ordering}

In a series of papers, we presented a number of analytic solutions that give the coherent transition probabilities of threelevel systems driven by two lasers of various pulse shapes and spatial separations. A remarkable result emerges from these analytic results. To achieve an efficient transfer of population from state 1 to state 3 that would not be sensitively dependent on the input parameters such as the laser pulse shapes, laser intensities and frequency modulation, the atoms or molecules should interact first with the laser pulse for the 2-3 transition and then with the laser pulse for the 1-2 transition, the pulse being overlapped in 
time. That is, a counterintuitive procedure is suggested. An important feature of this process is that as the pulse amplitudes are increased, the maximum occupation probability of the intermediate state 2 decreases rapidly. This is true even though the two laser frequencies are kept at one-photon resonance with the level transition frequencies $1-2$, and $2-3$ respectively. The counterintuitive interaction sequence makes it possible to get a complete transfer of population from state 1 to 3 without significantly populating state 2 . The high efficiency of this counterintuitive procedure has been strikingly demonstrated experimentally by Prof. K. Bergmann and his group at the University of Kaiserslautern who used two overlapping lasers to selectively populate high vibrational states of sodium molecules via an intermediate electronic state. Stimulated by the experimental work of Prof. Bergrnann, Prof. J. Reuss and his collaborators applied the counterintuitive two-laser excitation to $\mathrm{C}_{2} \mathrm{H}_{4}$ molecules with a similar success, and extended their work to infrared multiphoton excitation of $\mathrm{SF}_{6}$.

A potentially important application of the counterintuitive method of excitation is in the field of atomic interferometry, as suggested recentl by Marte, Zoller and Hall. Experimental observations of atomic interference fringes by scattering atoms from mechanical gratings or by applying a sequence of short $\pi / 2, \pi$ and $\pi / 2$ laser pulses to atoms have been previously reported. On the other hand, consider atomic-beam deflection by adiabatic passage jetween Zeeman ground levels via Raman transitions induced by counterpropagating $\sigma^{ \pm}$-polarized lasers arranged in the counterintuitive interaction sequence. Complete population transfer between the ground states can be achieved which corresponds to the scattering of the atornic wave packet into a single final momentum state by absorption and induced emission of laser photons. A distinctive advantage of this counterintuitive process is that although the lasers can be resonant, the excited (intermediate) states are never populated during the adiabatic transfer. The process should thus be insensitive to the laser intensities or to the effects of spontaneous emission, and thus preserves the coherence of the atomic wave function. 
publications (1982-1990)

1. F.T. Hioe

Exact Solitary Wave Solution of Short Different Wavelength optical fulses in $\mathrm{N}$-Level Atomic Systems Phys. Rev. A26, 1466 (1982)

2. F.T. Hioe and J.H. Eberly Nonlinear Constants of Motion for Three-Level Quantum Systems

Phys. Rev. A25, 2168 (1982)

3. F.T. Hioe, H.I. YoO and J.H. Everly

Statistical Analysis of Long-Term Dynamic Irregularity in an Exactly Soluble Quantum Mechanical Model

Mathematics studies 80,95 (1983), North-Holland Publ. Co.

4. F.T. Hioe

on the Mean Motion of Some statistical properties of a Quasiperiodic Observable in a Fermion-Boson Model

J. Math. Phys. 23, 2430 (1982)

5. F.T. Hioe

On Pearson's Random Walk and some statistical

Properties of a Quasiperiodic observable in a

Simple Quantum Model

J. Stat. Phys. 30, 467 (1983)

6. F.T. Hioe

Dynamic Symmetries in Quantum Electronics

Phys. Rev. A28, 879 (1983)

7. F.T. Hioe

Theory of Generalized Adiabatic Following in Multi-Level

sytems

Phys. Lett. 99A, 150 (1983)

8. F.T. Hioe

Theory of Atomic Excitation by Multiple Laser Pulses Proceeding of the 5 th Rochester conference in Quantum optics Edited by $I$. Mandel and E. Wolf, Plenum Press, New York, 1984 , p. 965.

9. J. Oreg, F.T. Hioe and J.H. Eberly

Adiabatic population Inversion in Multilevel systems proceeding of the 5th Rochester Conference in Quantum optics Edited by $L$. Mandel and E. Wolf, Plenum Press, New York, 1984, p. 985.

10. F.T. Hioe and J.H. Eberly

Multiple Laser Excitation on Multilevel Atoms

Phys. Rev. A29, 1164 (1984) 
11. J. Oreg, F.T. Hioe and J.H. Eberly

Adiabatic Following in Multilevel systems

Fhys. Rev. A29, 690 (1984)

12. F.T. Hioe

Linear and Nonlinear Constants of Motion for Three-Level Quantum Systems

Phys. Rev. A29, 3434 (1984)

13. F.T. Hioe

Solution of Bloch Equations Involving Amplitude and Frequency Molulations

Phys. Rev. A30, 2100 (1984)

14. F.T. Hioe

Analytic solutions of Density Matrix Evolutions with the Use of Racah Tensorial Decompositions

Phys. Rev. A30, 3097 (1984)

15. F.T. Hioe and C.E. Carroll

Analytic Solutions to the Two-state Problems for Chirped Pulses

J. Opt. Soc. Amer, B2, 497 (1985)

16. F.T. Hioe and C.E. Carroll

Two-state Problems Involving Arbitrary Amplitude and Frequency Modulations

Phys. Rev. A 32,1541 (1985)

17. F.T. Hioe

Gell-Mann Dynamic Symmetry for N-Level Quantum systems Phys. Rev. A32, 2824 (1985)

18. C.E. Carroll and F.T. Hioe

Further Generalization of Landau-Zener Calculation

J. Opt. Soc. Amer. B2, 1355 (1985)

19. F.T. Hioe

Some statistical and Dynamical problems in Quantum

Electronics

"The Wonderful World of Stochastics, A Tribute to Elliott W. Montroll", Edited by M.F. Shlesinger and G.H. Weiss, Elsevier Science Publishers, B.V. 1985

20. C.R. Carroll and F.T. Hioe

Generalization of the Landau-zener Calculation to Three Levels

J. Phys. A19, 1151 (1986) 
21. C.E. Carroll and F.T. Hioe

Transition Probabilities for the Three-Level Landau-Zener Model

J. Phys. A19, 2061 (1986)

22. X.W. Wang, D.L. Iin and F.T. Hioe

Mean Switching Time of Bistable Two-Mode Lasers

Phys. Rev. A34, 1251 (1986)

23. C.E. Carroll and F.T. Hioe

A New Class of Analytic Solutions of the Two-Stae Problem J. Phys. A19, 3579 (1986)

24. Z. Deng and F.T. Hioe

Chaos-Order-Chaos Transitions in a Two-Dimensional

Hamiltonian System, Phys. Rev. Lett. 55, 1539 (1985), 56, 1757 (1986) (E)

25. Z. Deng and F.T. Hioe

Stability-Instability Transitions in a system of Nonlinearly Coupled Harmonic oscillators

Phys. Lett. A115, 21 (1986)

26. F.T. Hioe and $Z$. Deng

Analytic solution of stability-Instability Transitions in a Two-Dimensional Hamiltonian System

Phys. Rev. A34, 3539 (1986)

27. C.E. Carroll and F.T. Hioe

Three-state Model Driven by Two Laser Beams

Phys. Rev. A36, 724 (1987)

28. F.T. Hioe and $\mathrm{Z}$. Deng

Stability-Instability Transitions in Hamiltonian systems of n Dimensions

Phys. Rev. A35, 847 (1987)

29. F.T. Hioe

N-Level Quantum Systems with SU(2) Dynamic symetry

J. Opt. Soc. Am. B4, 1327 (1987)

30. C.E. Carroll and F.T. Hioe

Driven Three-state Model and Its Analytic Solutions

J. Math. Phys. 29, 487 (1988)

31. F.T. Hioe

N-Level Quanturn Systems with Gell-Mann Dynamic Symmetry

J. Opt. Soc. Am. B, 859 (1988)

32. F.T. Hioe and C.E. Carroll

Coherent population Trapping in $\mathrm{N}$-Level Quancum Systems

Phys. Rev. A37, 3000 (1988) 
33. C.E. Carroll and F.T. Hioe

Three-state Systems Driven by Resonant optical Pulses of Different Shapes

J. Opt. Soc. Am. B5, 1335 (1988)

34. F.T. Hioe

Hamiltonian Systems with a Certain stability Property

J. Phys. A21, L377 (1988)

35. F.T. Hioe

Certain Stability Type and Integrable Two-Dimensional

Hamiltonian systems

Phys. Rev. A39, 2628 (1989)

36. F.T. Hioe

Analytic solutions and Dynamic symmetries in Laser-Driven Atomic Excitation

"Lasers, Molecules and Methods", edited by J.O.

Hirschfelder, R.E. Wyatt and R.D. Coalson, John wiley \& Sons, Inc. 1989

37. F.T. Hioe

Lossless Propagation of Optical Pulses Through N-Level Systems With SU(2) symmetry

J. Opt. Soc. Am. BE, 335 (1989)

38. F.T. Hioe

Lossless Propagation of Optical Pulses Through N-Level Systems with Gell-Mann symmetry

J. Opt. Soc. Am. BE, 1245 (1989)

39. C.E. Carroll and F.T. Hice

Two-Photon Resonance in Three-state Model Driven by Two Laser Beams

J. Phys. B2L, 2633 (1989)

40. T.T. Hioe

Grouping of Products of Wavefunctions and Gell-Mann Dynamic Symmetry

J. Phys. A22, 4143 (1989)

41. J.R. Ruklinski, U. Gaubatz, F.T. Hioe and K. Bergmann Adiabatic Population Transfer in a Three-Level System Driven by Delayed Laser Pulses

Phy. Rev. A 40,6741 (1989)

42. F.T. Hioe, Restricted Walks, Stability-Instability Transitions, and Dynamic Symmetries

J. Stat. Phys. 58, 627 (1990)

43. C.E. Carroll and F.T. Hioe

Another Analytic Solution of the Two-state Problem Phys. Rev. A41, 2835 (1990) 
44. C. E. Carroll and F. T. Hioe Analytic Solutions for Three-State Systems with Overlapping Pulses Phys. Rev. A42, 1522 (1990)

45. F. T. Hioe and C. E. Carro.1 Excitation of Three-State Atom with Minimum Population of Intermediate State Coherence and Quantum Optics VI, Edited by J. H. Eberly, L. Mandel and E. Wolf, Plenum Press, New York 1990, p. 493 

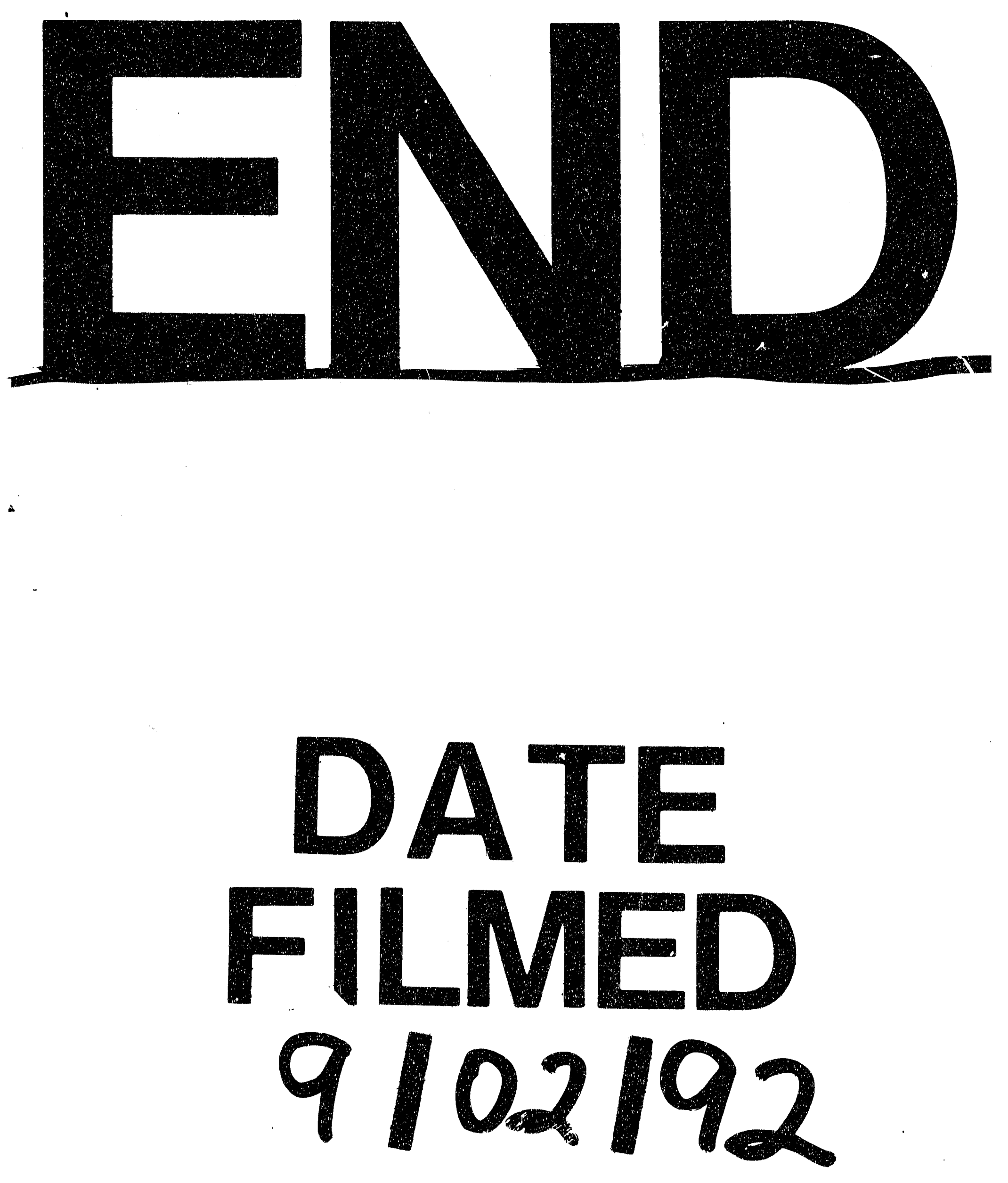